\title{
Correction to: Site amplification in the Kathmandu Valley during the 2015 M7.6 Gorkha, Nepal earthquake
}

\section{S. Tallett-Williams ${ }^{1} \cdot$ B. Gosh ${ }^{2}$. S. Wilkinson ${ }^{3} \cdot$ C. Fenton ${ }^{4} \cdot$ P. Burton ${ }^{5} \cdot$ M. Whitworth ${ }^{6}$.

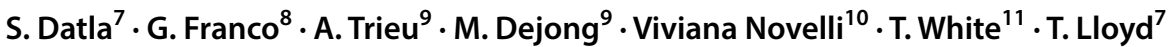

\section{Correction to: Bull Earthquake Eng (2016) 14:3301-3315 https://doi.org/10.1007/s10518-016-0003-8}

This erratum is published as author name was published as V. Novellis and needs to be correctly read as Viviana Novelli.

Publisher's Note Springer Nature remains neutral with regard to jurisdictional claims in published maps and institutional affiliations.

The original article can be found online at https://doi.org/10.1007/s10518-016-0003-8.

S. Tallett-Williams

sarah.tallett-williams09@imperial.ac.uk

1 Imperial College London, South Kensington Campus, London SW7 2AZ, UK

2 Mott McDonald, Croydon, UK

3 Newcastle University, Newcastle upon Tyne, UK

4 University of Canterbury, Christchurch, New Zealand

5 University of East Anglia, Norwich, UK

6 AECOM, London, UK

7 AIR Worldwide, London, UK

8 Guy Carpenter, London, UK

9 University of Cambridge, Cambridge, UK

10 University College London, London, UK

11 ARUP, London, UK 